\title{
Self-quotient Referential Bilateral Filter and Its Application to Real-Time Face Beautification System
}

\author{
M. Matsumoto* \\ University of Electro-Communications, 1-5-1, Chofugaoka, Chofu-shi, Tokyo, Japan. \\ * Corresponding author. Email: mitsuharu.matsumoto@ieee.org \\ Manuscript submitted August 6, 2014; accepted January 13, 2015. \\ doi: 10.17706/ijcee.2015.v7.875
}

\begin{abstract}
In this paper, self-quotient referential bilateral filter is introduced. Although bilateral filter is a well-known edge-preserving smoothing filter, it is difficult to handle with light variation. The proposed filter combines bilateral filter and self-quotient filter, and can handle the image with light variation. In our method, we utilize self-quotient image as a reference filter for bilateral filter. Some experimental results are shown to demonstrate the effectiveness of the proposed method. As an application, we also developed a real-time face beautification system combining face detector and the proposed filter. Throughout the experiments, our method has a potential to be used for real-time face beautification system.
\end{abstract}

Key words: Bilateral filter, edge-preserving filter, self-quotient filter, self-quotient referential bilateral filter.

\section{Introduction}

"Keeping youth". Although it is an important wish of humans, it is difficult for us to stop physical deterioration.

Wrinkles and age spots appear on face with age, and they may prevent old people to communicate with each other.

We may help to give motivation to elderly people if we can reduce such wrinkles and age spots on their faces by using face beautification system. For instance, young-looking photographs and television-phone system are examples of applications of face beautification system.

As wrinkles and age spots on our face are basically small cracks and noise on face, filtering for edge-preserving smoothing is a solution to realize face beautification.

Although many studies on edge-preserving smoothing have been proposed in the pasts [1]-[5], bilateral filter is a promising filter for this purpose [6]-[7].

The algorithm of bilateral filter is very simple, and only two parameters are required. One is the parameter for reducing the effect of the pixel far from the processed pixels. The other is the parameter for reducing the effect of the intensity whose distance from the processed pixel is large. The procedure of bilateral filter can be processed non-iteratively.

Although bilateral filter is a useful filter as described above, it is difficult to handle the image which not only has a high contrast area but also has a low contrast area. This is because bilateral filter filters the image based on the local intensity of the image to preserve edge.

Using two images taken in different situation is a typical approach to solve this problem. The method using flash and non-flash images is reported by Eisemann et al. [8]. The main idea of Eisemann algorithm is 
to use flash image for detecting the details of the image while to use non-flash image for smoothing image based on the information of flash image. Bennett et al. also reported the algorithm of multispectral fusion using the image which is taken under visible light condition and that was taken under infra-red light [9].

These approaches require two different types of images taken under different condition at the same time to avoid motion of the photographer or/and objects.

To solve the problems, we employ self-quotient filter and proposed self-quotient referential bilateral filter [10].

Self-quotient filter is a filter to extract edge information of the image which not only has high contrast area but also has low contrast area [11], [12]. By dividing the original image by its blurred image, self-quotient filter can extract the edge information regardless of the contrast in the image.

The filter is the extended version of quotient image method [13], [14]. Self-quotient filter is defined as the ratio of the original image and its smoothed image. It requires only an image and can extract edge information not only around high contrast area but also around low contrast area.

Self-quotient referential bilateral filter is an improved bilateral filter which refers to self-quotient image instead of the original image in bilateral filter. By using self-quotient image, we can preserve edge not only in high contrast area but also in low contrast area.

Although self-quotient referential bilateral filter can handle the image with light variation, it cannot handle a color image. Hence, in this paper, we improve the filter for color images. As an application of our system, we also implement the proposed filter to the real-time face beautification system, and show the results.

\section{Algorithm}

In this section, the proposed algorithm is described. Fig. 1 shows the flow of our method.

Let $x(\mathbf{i})=x\left(i_{1}, i_{2}\right)$ be the color input image. In $Y C r C b$ space, $x\left(i_{1}, i_{2}\right)$ can be described as

$$
x\left(i_{1}, i_{2}\right)=\left(x_{Y}\left(i_{1}, i_{2}\right), x_{C r}\left(i_{1}, i_{2}\right), x_{C b}\left(i_{1}, i_{2}\right)\right)
$$

where $x_{Y}\left(i_{1}, i_{2}\right), x_{C r}\left(i_{1}, i_{2}\right)$, and $x_{C b}\left(i_{1}, i_{2}\right)$ represent the intensity of the image, the value of $C b$ color space and $\mathrm{Cr}$ color space at position $\mathbf{i}=\left(i_{1}, i_{2}\right)$, respectively. Let us define $F(x)$ as the output of the filter $F$ applied to the image. Let $S$ be the set of all possible image locations. $|\cdot|$ and $\|\cdot\|$ is defined as absolute norm and $L_{2}$ norm, respectively. For example, $\|\mathbf{i}-\mathbf{j}\|$ represents the Euclidean distance between pixels $\mathbf{i}$ and $\mathbf{j}$.

Let us start a simple blurring using Gaussian filter.

Gaussian filter is defined as follows:

$$
G F(x(\mathbf{i}))=\sum_{\mathbf{j} \in S} G_{\sigma}(\|\mathbf{i}-\mathbf{j}\|) x(\mathbf{j})
$$

where $G_{\sigma}(x)$ is two dimensional Gaussian described as follows:

$$
G_{\sigma}(x)=\frac{1}{2 \pi \sigma^{2}} \exp \left(-\frac{x^{2}}{2 \sigma^{2}}\right)
$$

Gaussian filter is a weighted average filter whose impulse response is Gaussian function. The filter weight is defined by the Gaussian $G_{\sigma}(\|\mathbf{i}-\mathbf{j}\|)$. In Gaussian filter, $\sigma$ is a parameter to control how far the neighborhood should be considered.

Due to this design, Gaussian filter depends only on the spatial distance between the processed pixel and 
the neighbor pixels. It does not consider their values. Hence, in Gaussian filter, if bright pixels and dark pixels are close to each other, the area is strongly blurred.

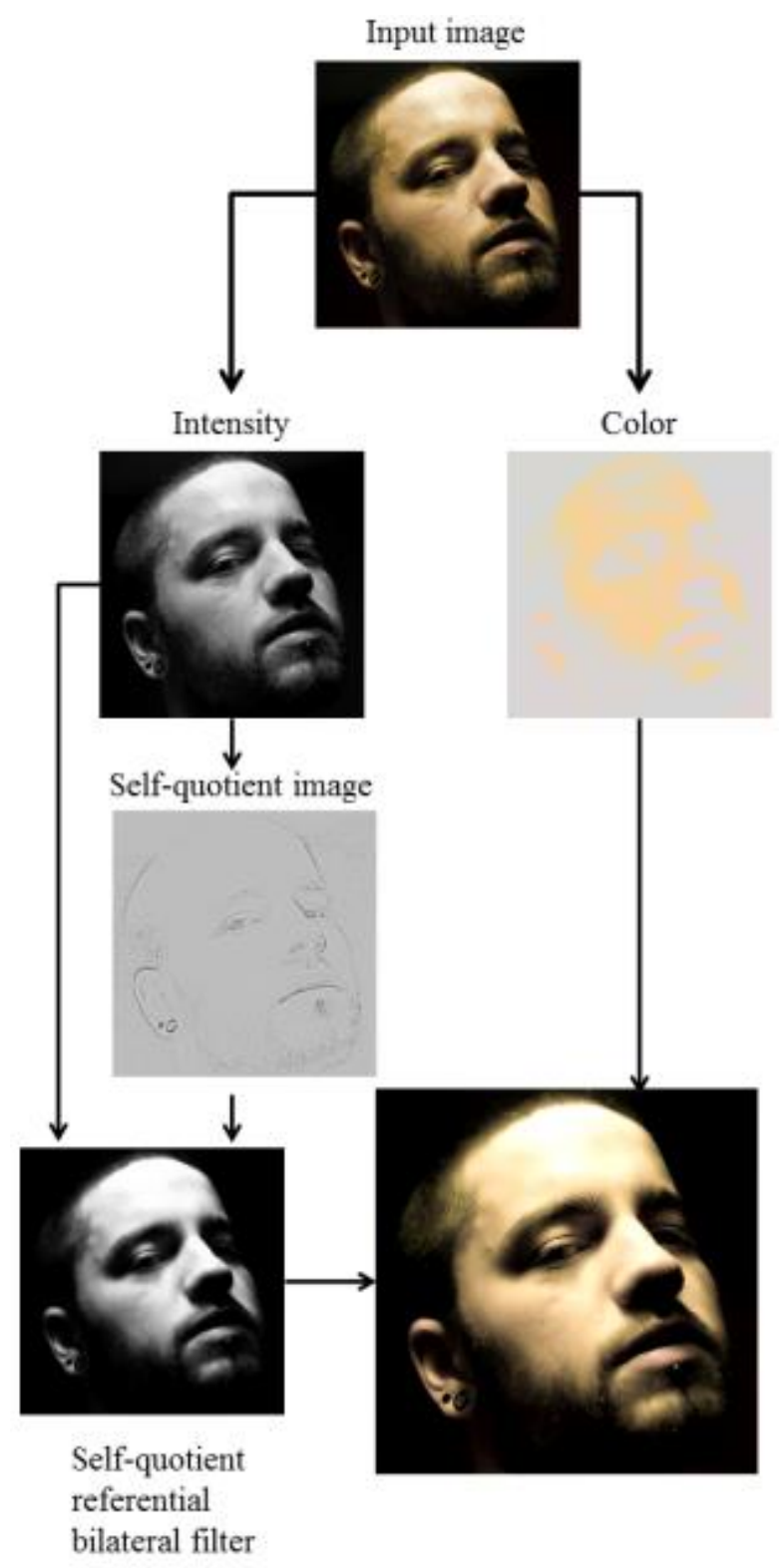

Fig. 1. Flow of the proposed algorithm.

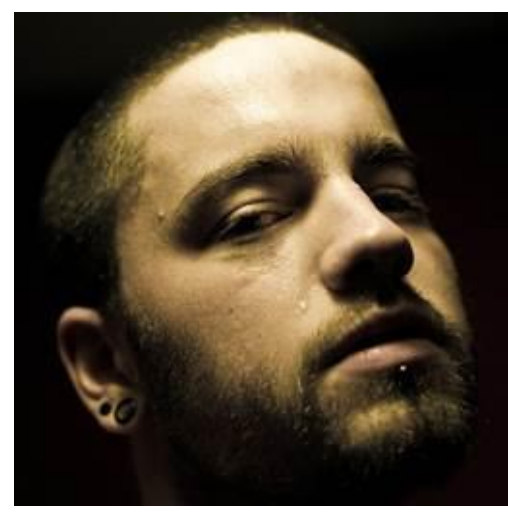

(a) Input image

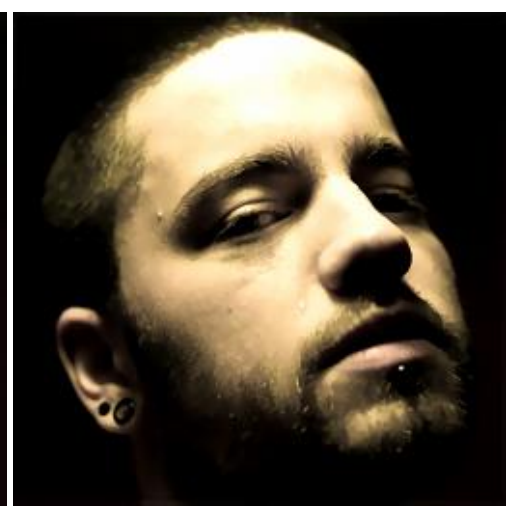

(b) Example 1

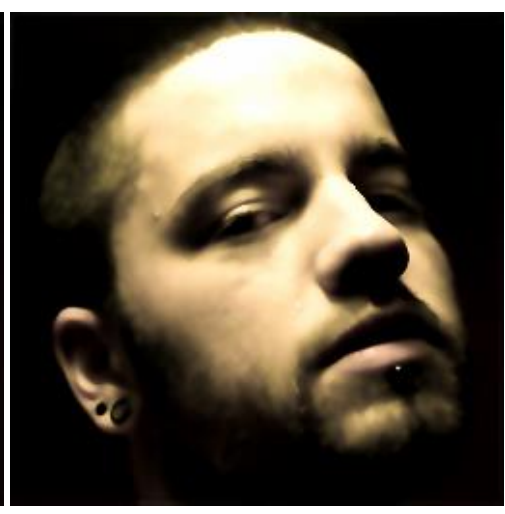

(c) Example 2

Fig. 2. Examples of bilateral filter. 
Bilateral filter aims to solve this problem by considering not only the spatial distance between the processed pixel and the neighbor pixels, but also the intensity difference between the processed pixel and the neighbor pixels.

Bilateral filter (BF) can be described as follows:

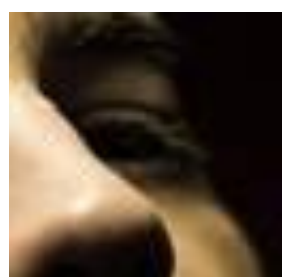

(a) Input image

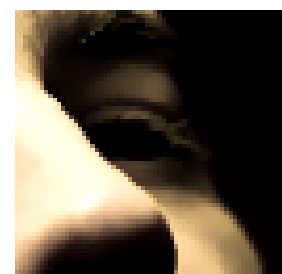

(b) Example 1

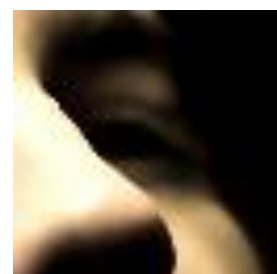

(c) Example 2

Fig. 3. Closeup images of bilateral filter.

$$
B F(x(\mathbf{i}))=\frac{1}{W_{\mathbf{i}}} \sum_{\mathbf{j} \in S} G_{\sigma_{s}}(\|\mathbf{i}-\mathbf{j}\|) G_{\sigma_{r}}(\|x(\mathbf{i})-x(\mathbf{j})\|) x(\mathbf{j})
$$

where $W_{\mathrm{i}}$ represents the normalization factor described as follows:

$$
W_{\mathbf{i}}=\sum_{\mathbf{j} \in S} G_{\sigma_{s}}(\|\mathbf{i}-\mathbf{j}\|) G_{\sigma_{r}}(\|x(\mathbf{i})-x(\mathbf{j})\|)
$$

Parameter $\sigma_{s}$ is a parameter to consider spatial distance between the processed pixel and the neighbor pixels. Parameter $\sigma_{r}$ is a parameter to consider intensity difference between the processed pixel and the neighbor pixels. If $\sigma_{r}$ becomes larger, bilateral filter becomes similar to Gaussian filter. As described above, bilateral filter uses intensity difference between the processed pixel and the neighbor pixels to preserve edge information.

Due to the design, it is difficult for bilateral filter to handle the image taken under varying lighting condition.

Fig. 2 shows examples to clarify this point. Fig. 2(a) shows the input image. As shown in Fig. 2(b), if the smoothing effect of bilateral filter is small, the smoothing is insufficient although the edge information around low contrast area is preserved. On the other hand, as shown in Fig. 2(c), if the smoothing effect of bilateral filter is large, the edge information around low contrast area is blurred, while the smoothing is sufficient. Fig. 3 is the closeup image around left eye. As shown in Fig. 3(c), the eye area is blurred.

Although bilateral filter can smooth the image while preserving edge information in high contrast area, the edge in low contrast area is blurred.

Cross bilateral filter is an improved bilateral filter, and uses two images to solve this problem. One is a flash image, the other is a non flash image. In cross bilateral filter, a flash image is used to extract the details of the image, and non-flash image is filtered not by using non-flash image but by using a flash image as a reference.

When we take a photograph with non-flash, the image texture is natural but image includes high-level noise. Cross bilateral filter gives a solution to this problem by using a flash image.

Let $F$ and $N$ be a flash image and a non-flash image at the same place, respectively. Cross bilateral filter $(\mathrm{CBF})$ is defined as follows:

$$
C B F(x(\mathbf{i}))=\frac{1}{W_{\mathbf{i}}} \sum_{\mathbf{j} \in S} G_{\sigma_{s}}(\|\mathbf{i}-\mathbf{j}\|) G_{\sigma_{r}}(\|F(\mathbf{i})-F(\mathbf{j})\| N(\mathbf{j})
$$


Although cross bilateral filter can output a natural image, it requires two images taken at the same position.

We aim to obtain such natural image by using an image.

To achieve this aim, we utilize self-quotient image instead of flash image.

Self-quotient filter (SQF) is a simple filter to extract edge information from an image with varying light condition. It can be described as follows:

$$
\operatorname{SQF}(x(\mathbf{i}))=\frac{x(\mathbf{i})}{G_{\sigma_{t}}(x(\mathbf{i}))}
$$

where parameter $\sigma_{t}$ is to design how far the neighbor pixels should be considered. Due to the self-quotient effect of self-quotient filter, we can extract the texture and edge information from an image taken under varying light condition.

Self-quotient referential bilateral filter (SRBF) is described as follows:

$$
\operatorname{SRBF}(x(\mathbf{i}))=\frac{1}{W_{\mathbf{i}}} \sum_{\mathbf{j} \in S} G_{\sigma_{s}}(\|\mathbf{i}-\mathbf{j}\|) G_{\sigma_{r}}(\|S Q F(x(\mathbf{i}))-\operatorname{SQF}(x(\mathbf{j}))\|) x(\mathbf{j})
$$

where $W_{\mathrm{i}}$ is expressed as follows:

$$
W_{\mathbf{i}}=\sum_{\mathbf{j} \in S} G_{\sigma_{s}}(\|\mathbf{i}-\mathbf{j}\|) G_{\sigma_{r}}(\|S Q F(x(\mathbf{i}))-S Q F(x(\mathbf{j}))\|)
$$

SRBF can smooth the image while preserving edge not only around high contrast area but also low contrast area because it refers the filter output of self-quotient filter instead of the original image unlike bilateral filter.

As the filter output of self-quotient filter can be generated from an original image, SRBF only requires an image. Let us confirm the effectiveness of our approach through some experiments in the next section.

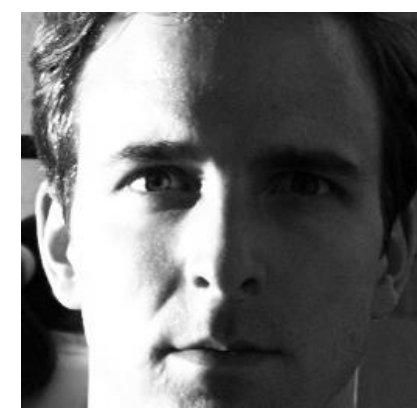

(a) Input image

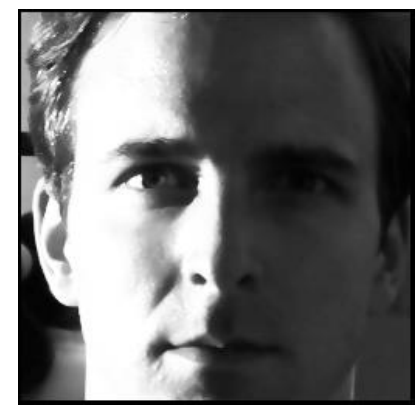

(b) Bilateral filter

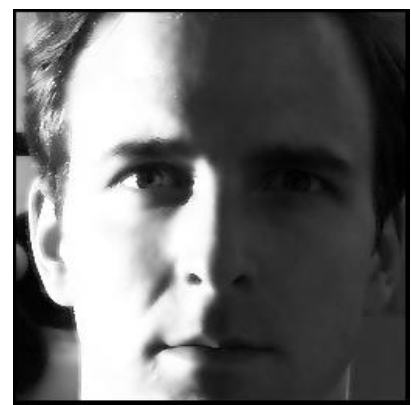

(c) Proposed filter

Fig. 4. Comparison of bilateral filter and self-quotient referential bilateral filter (The image was selected from Yale image database. The file name is yaleB10_P00A+095E+00.pbm).

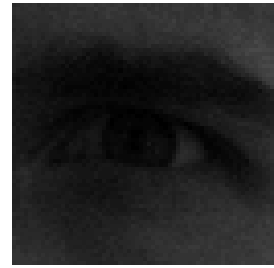

(a) Input image

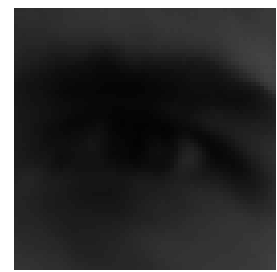

(b) Bilateral filter

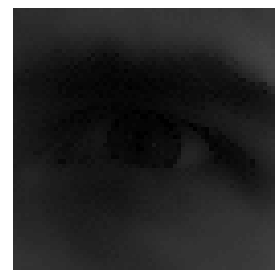

(c) Proposed filter

Fig. 5. Comparison of bilateral filter and self-quotient referential bilateral filter (Closeup image from eye area. The image was cut from yaleB10_P00A+095E+00.pbm). 


\section{Experiments}

\subsection{Experiments on Gray and Color Images}

We conducted some experiments to check the effectiveness of our approach. The results of gray scale case are shown at first. Some images were selected from Yale image database [15] and cut face area from them. The image size is 256 pixels $\times 256$ pixels. We used a laptop computer whose CPU was Intel Core i5-3210M $2.50 \mathrm{GHz}$. The programs were implemented by MATLAB.

Fig. 4 shows the filter output of bilateral filter and self-quotient referential bilateral filter.

Fig. 4(a) shows the input image, whose file name is yaleB10_P00A+095E+00.pbm.

Fig. 4(b) and Fig. 4(c) show the filter output of biletaral filter and self-quotient referential bilateral filter, respectively.

To clarify the problem of bilateral filter, we also show the closeup image around left eye area in Fig. 5 .

As shown in Fig. 4 and Fig. 5, when the bilateral filter was applied, the edge around right eye area could be preserved while smoothing surface roughness. However, the left eye area was blurred when bilateral filter was applied because the left eye area was low contrast.

On the other hand, we could smooth the image while preserving the edge not only around the right eye but also around the left eye in the case of the proposed filter.

We also show another example in Fig. 6 and Fig. 7.

As shown in Fig. 6 and Fig. 7, better performance could be obtained when we utilized the proposed filter.

We next show the results of color images.

The images were selected from the Internet. The images are licensed with creative commons license.

The image reference is shown in figure caption. Fig. 8 shows the results of the experiment. Fig. 9 shows the closeup image of the left eye. As shown in Fig. 8 and Fig. 9, when the bilateral filter was employed, the edge around left eye was blurred similar to the results of gray scale images.

Fig. 10 and Fig. 11 show another example of color images.

As shown in Fig. 10 and Fig. 11, better performance could be obtained when we utilized the proposed filter.

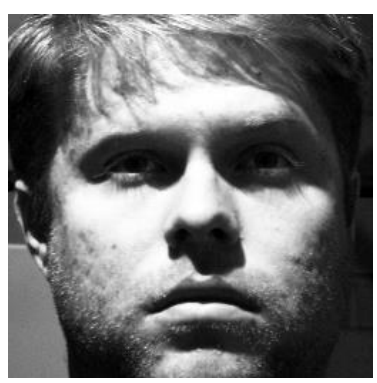

(a) Input image

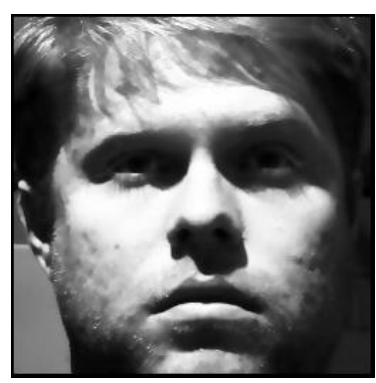

(b) Bilateral filter

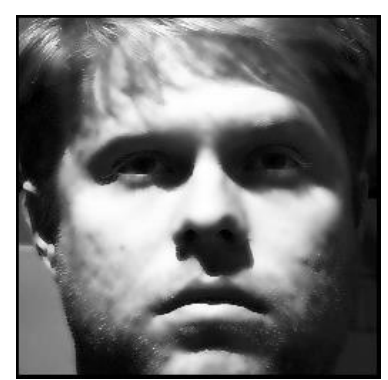

(c) Proposed filter

Fig. 6. Comparison of bilateral filter and self-quotient referential bilateral filter (The image was selected from Yale image database. The file name is yaleB01_P01A+035E+65.pbm. )

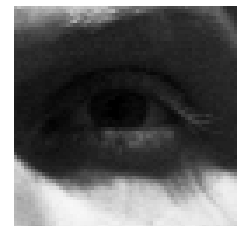

(a) Input image

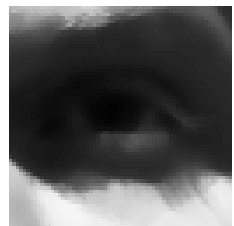

(b) Bilateral filter

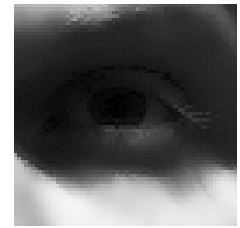

(c) Proposed filter

Fig. 7. Comparison of bilateral filter and self-quotient referential bilateral filter (Closeup image from eye area. The image was cut from yaleB10_P00A+095E+00.pbm). 


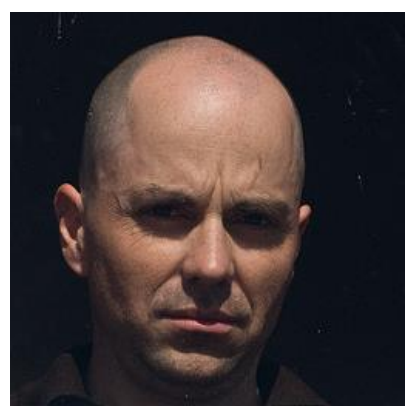

(a) Input image

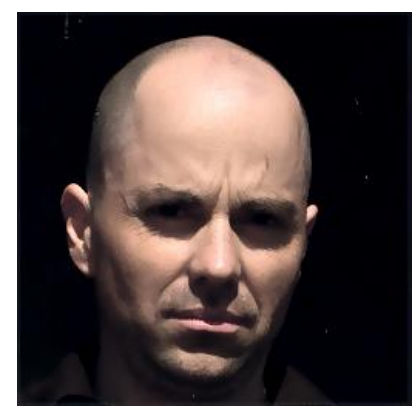

(b) Bilateral filter

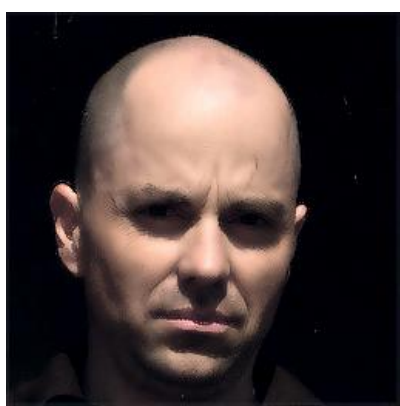

(c) Proposed filter

Fig. 8. Comparison of bilateral filter and self-quotient referential bilateral filter (The image was selected from in http://www.flickr.com/photos/jonwiley/2109674871/lightbox/ (Photograph by Jon Wiley)).

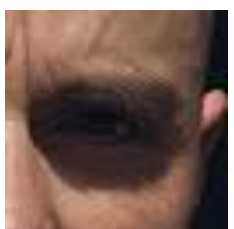

(a) Input image

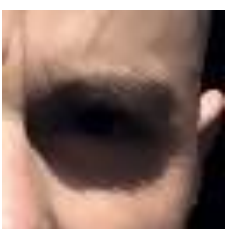

(b) Bilateral filter

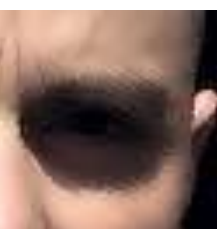

(c) Proposed filter

Fig. 9. Comparison of bilateral filter and self-quotient referential bilateral filter (Closeup image from eye area.)

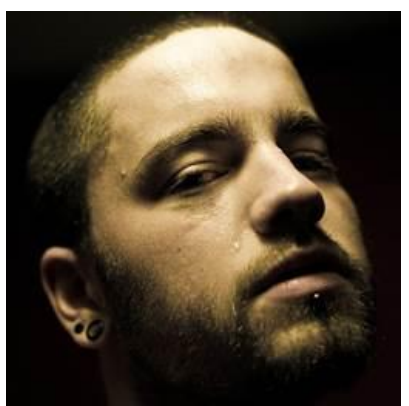

(a) Input image

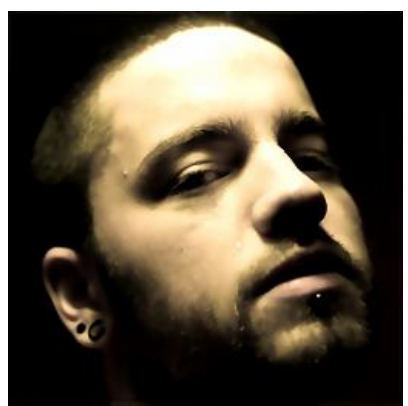

(b) Bilateral filter

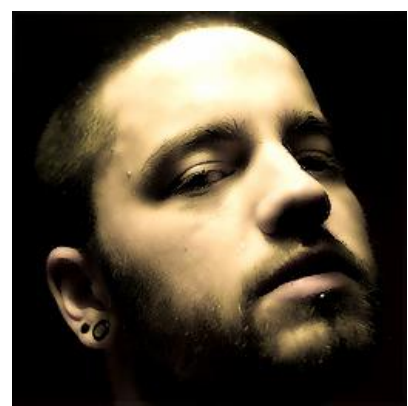

(c) Proposed filter

Fig. 10. Comparison of bilateral filter and self-quotient referential bilateral filter (The image was selected from in http://www.flickr.com/photos/ch-ed/4255702269/lightbox/ (Photograph by Ch. Ed)).

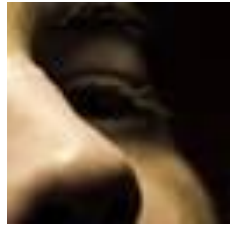

(a) Input image

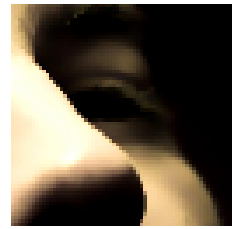

(b) Bilateral filter

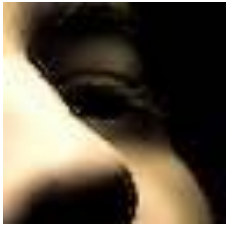

(c) Proposed filter

Fig. 11. Comparison of bilateral filter and self-quotient referential bilateral filter (Closeup image from eye area.)

\subsection{Real Time System Implementation}

We also implemented real-time face beautification system.

The same laptop computer was used for implementation. The development software is Visual studio 2010. Visual C\# was used for implementation. OpenCV2.4.5 and OpenCVSharp were used for image processing. 
The implemented systems are shown in Fig. 12.

As shown in Fig. 12, we used Haar cascade for face detection after capturing the image with web camera.

We then apply the proposed filter to the detected area, and combine the filter output and the captured image.

After face detection, we apply self-quotient referential bilateral filter to the detected area. The face area was surrounded by the red square to clarify the detected area.

For testing, we prepared three old man/woman photographs and capture them using our developed system. Fig. 13, Fig. 14, and Fig. 15 show the detected and filtered results.

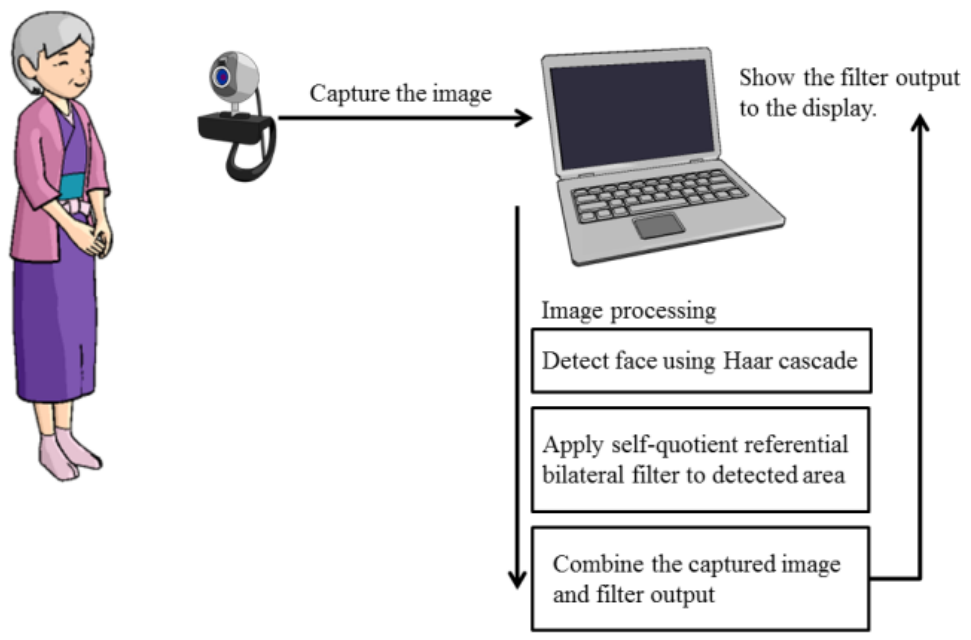

Fig. 12. Conceptual diagram of real-time face beautification system.

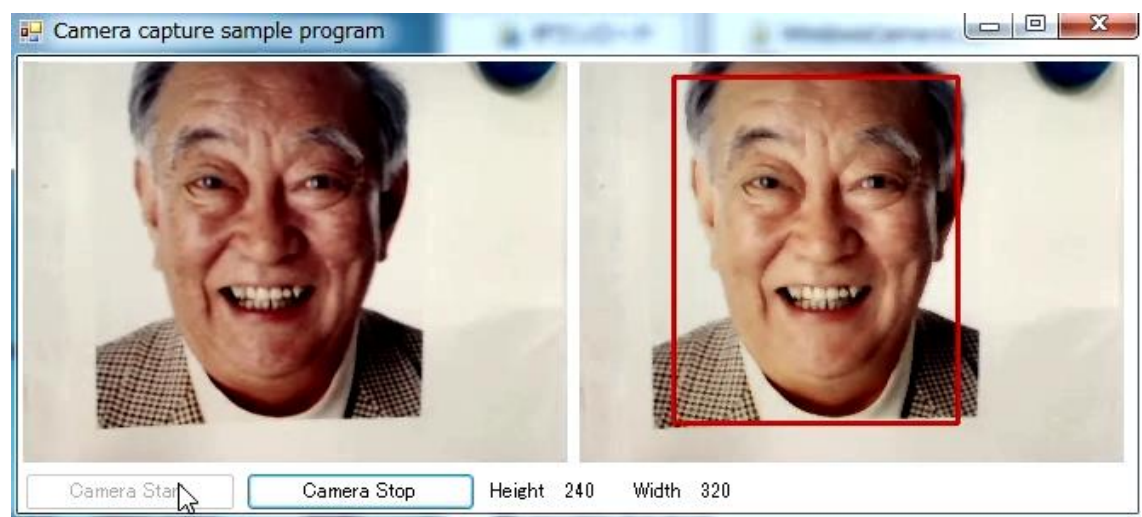

Fig. 13. Example 1 of real-time face beautification system.

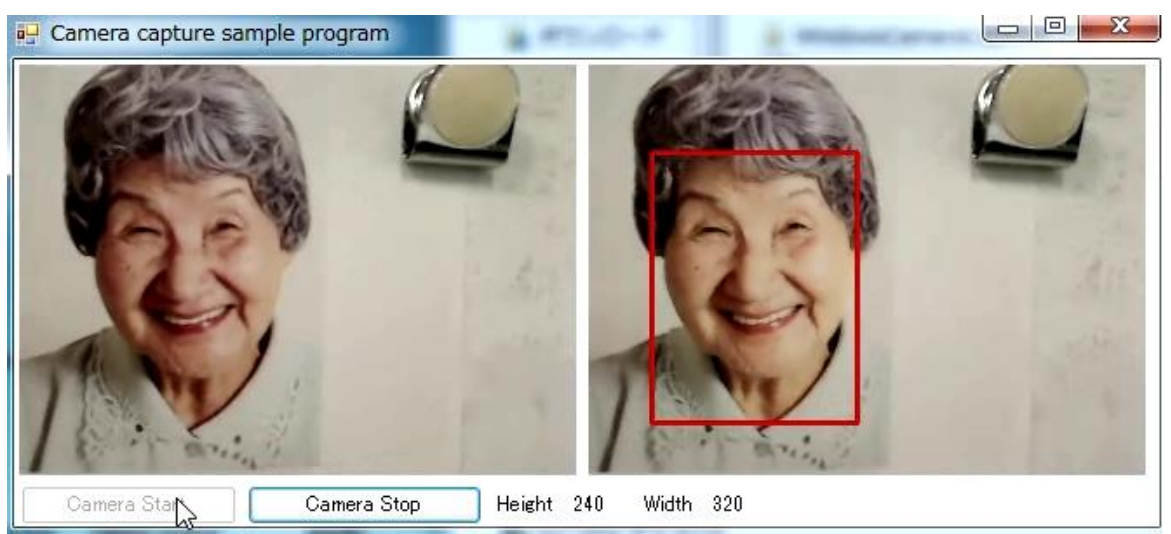

Fig. 14. Example 1 of real-time face beautification system. 


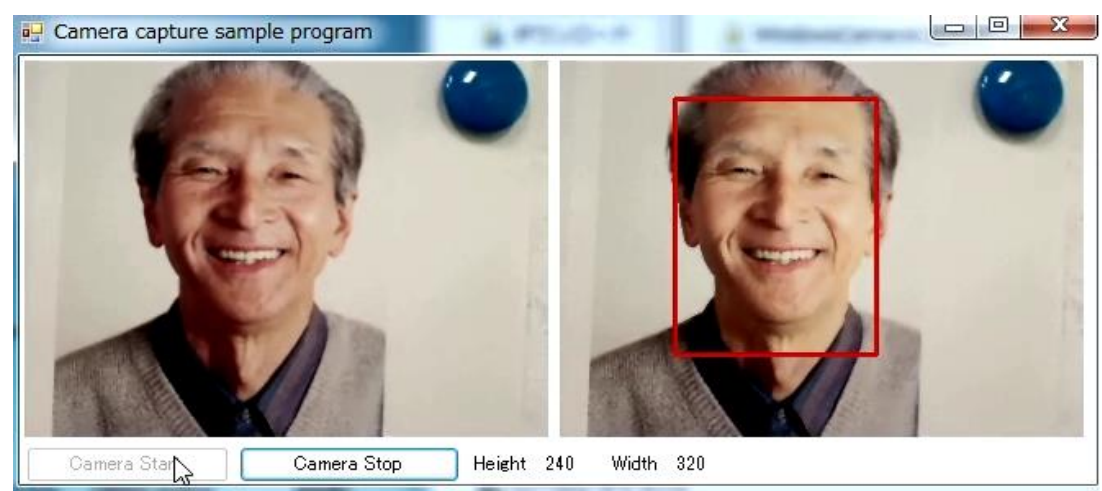

Fig. 15. Example 3 of real-time face beautification system.

In Fig. 13-Fig. 15, left pictures show the original images captured by web-camera. Right pictures show the outputs combining the original images and filter outputs.

As shown in Fig. 13-Fig. 15, the system could robustly detect the face area and smooth its face while preserving edge information.

\section{Discussion and Conclusion}

In this paper, we proposed self-quotient referential bilateral filter, and showed the real-time face beautification system using our system as an application of the proposed filter.

Although it is difficult to handle the image with light variation using original bilateral filter, the proposed filter could smooth while preserving edge information not only around high contrast area but also low contrast area.

If the method using flash and non-flash images is employed, it requires two images taken at the same place. Our system only requires an image because it refers to self-quotient image.

These features are preferable for applying our method to existing systems such as existing television phone system and TV programs. We would like to apply the developed system to existing systems. We also would like to conducted user experiment for subjective evaluation.

\section{Acknowledgment}

This work was supported by JSPS KAKENHI Grant Number 25730107, 2013. This work was also supported by Japan Prize Foundation, Foundation for the Fusion of Science and Technology and NS promotion foundation for science of perception.

\section{References}

[1] Pomalaza-Raez, C., \& McGillem, C. D. (1984). An adaptative, nonlinear edge-preserving filter. IEEE Trans. on Acoustics, Speech and Signal Processing, 32(3), 571-576.

[2] Boult, T., Melter, R. A., Skorina, F., \& Stojmenovic, I. (1993). G-neighbors. Proceedings of SPIE Conf. on Vision Geometry II (pp. 96-109).

[3] Arakawa, K., Matsuura, K., Watabe, H., \& Arakawa, Y. (2002). A method of noise reduction for speech signals using component separating-filters. IEICE Trans. on Fundamentals, J85-A(10), 1059-1069.

[4] Arakawa, K., \& Okada, T. (2005). Separating nonlinear filter bank and its application to face image beautification. IEICE Trans. on Fundamentals, 1216-1225.

[5] Himayat, N., \& Kassam, S. A. (1993). Approximate performance analysis of edge preserving filters. IEEE Trans. on Signal Processing, 41(9), 2764-2777.

[6] Tomasi, C., \& Manduchi, R. (1998). Bilateral filtering for gray and color images. Proceedings of Int'l Conf. 
on Computer Vision (pp. 839-846).

[7] Inoue, K., Ogawa, M., Hara, K., \& Urahama, K. (2010). Edge-preserving smoothing by iterative adaptive bilateral filter. IEICE Trans. on Information and Systems, J93-D(11), 2558-2560.

[8] Eisemann, E., \& Durand, F. (2004). Flash photography enhancement via intrinsic relighting. ACM Trans. on Graphics, 673-678.

[9] Bennett, E. P., Mason, J. L., \& McMillan, L. (2007). Multispectral bilateral video fusion. IEEE Transactions on Image Processing, 16(5), 1185-1194.

[10] Matsumoto, M. (2012). Self-quotient-referential bilateral filter for images under varying lighting conditions. Proceedings of the 38th Annual Conference of the IEEE Industrial Electronics Society (pp. 1570-1574).

[11] Wang, H., Li, S. Z., \& Wang, Y. (2004). Face recognition under varying lighting conditions using self quotient image. Proceedings of Int'l Conf. on Automation Face and Gesture Recognition (pp. 819-824).

[12] Wang, H., Zhang, J. J., Li, S. Z., \& Wang, Y. (2004). Shape and texture preserved non-photorealistic rendering. Computer Animation and Virtual Worlds, 15(3-4), 453-461.

[13] Shasua, A., \& Riklin-Raviv, T. (2001). The quotient image: Class-based re-rendering and recognition with varying illuminations. IEEE Trans. on Pattern Recognition and Machine Intelligence, 23(2), 129-139.

[14] Riklin-Raviv, T., \& Shashua, A. (1999). The quotient image: Class based recognition and systhesis under varying illumination. Proceedings of IEEE Conf. on Computer Vision and Pattern Recognition (pp. 566-571).

[15] Georghiades, A. S., Belhumeur, P. N., \& Kriegman, D. J. (2001). From few to many: Illumination cone models for face recognition under variable lighting and pose. IEEE Trans. Pattern Anal. Mach. Intelligence, 23(6), 643-660.

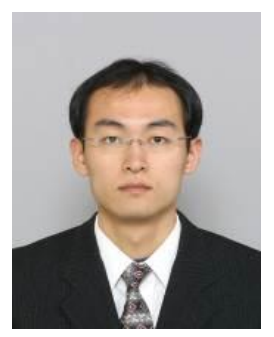

M. Matsumoto received a B.E. degree in applied physics, and a M.E. degree and a $\mathrm{PhD}$ degree of Eng. in pure and applied physics from Waseda University, Tokyo, Japan, in 2001, 2003 , and 2006, respectively.

$\mathrm{He}$ is currently an associate professor in the University of Electro-Communications. His research interests include array signal processing, blind source separation, image processing, optical device, pattern recognition, self-assembly and robotics. He received the Ericsson Young Scientist Award from Nippon Ericsson K.K, Japan and the FOST Kumada Award, in 2009 and 2011, respectively. He is a member of the Institute of Electrical and Electronic Engineers (IEEE). 